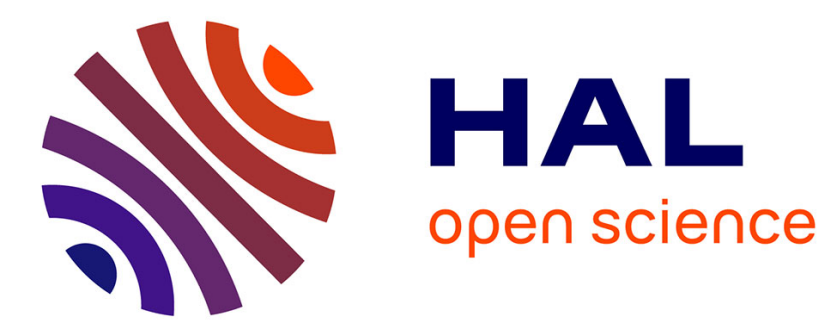

\title{
Structure des feuilletages kähleriens en courbure semi-négative.
}

Frédéric Touzet

\section{To cite this version:}

Frédéric Touzet. Structure des feuilletages kähleriens en courbure semi-négative.. Annales de la Faculté des Sciences de Toulouse. Mathématiques., 2010, 19 (3-4), pp.865-886. 10.5802/afst.1269 . hal-00407442

\section{HAL Id: hal-00407442 \\ https://hal.science/hal-00407442}

Submitted on 28 Aug 2009

HAL is a multi-disciplinary open access archive for the deposit and dissemination of scientific research documents, whether they are published or not. The documents may come from teaching and research institutions in France or abroad, or from public or private research centers.
L'archive ouverte pluridisciplinaire HAL, est destinée au dépôt et à la diffusion de documents scientifiques de niveau recherche, publiés ou non, émanant des établissements d'enseignement et de recherche français ou étrangers, des laboratoires publics ou privés. 


\title{
STRUCTURE DES FEUILLETAGES KÄHLERIENS EN COURBURE SEMI-NÉGATIVE
}

\author{
FRÉDÉRIC TOUZET
}

\begin{abstract}
RÉSUMÉ. Nous étudions dans cet article quelques propriétés des feuilletages (transversalement) kähleriens sur une variété compacte lorsque la forme de Ricci transverse est "suffisamment" négative. Nous établissons plus préscisément que l'algébre de Lie du pseudogroupe d'holonomie est semi-simple. Ceci fournit un critére qui assure que les feuilles d'un feuilletage holomorphe á classe canonique numériquement triviale sont fermées.
\end{abstract}

ABstract. This paper is concerned with (tranversally) Kähler foliations. We proved here that the holonomy pseudo-group's lie algebra is semi-simple under negativity assumptions of the transverse Ricci tensor. As a consequence, we obtain that the leaves of holomorphic foliations with trivial canonical class are closed submanifolds.

\section{NOTATIONS ET RAPPELS}

Soit $M$ une variété connexe munie d'un feuilletage régulier $\mathcal{F}$ de classe $\mathcal{C}^{\infty}$.

Désignons respectivement par $T M, T \mathcal{F}, \nu \mathcal{F}$ le fibré tangent de $M$, le fibré tangent et normal du feuilletage (identifié á un supplémentaire de $T \mathcal{F})$ et par $T_{\mathbb{C}} M, T_{\mathbb{C}} \mathcal{F}, \nu_{\mathbb{C}} \mathcal{F}$ leurs complexifiés respectifs.

On obtient ainsi une décomposition du fibré en algèbre extérieure

$$
\Lambda^{i} T_{\mathbb{C}}^{*} M=\bigoplus_{r+s=i} \Lambda^{r, s} T_{\mathbb{C}}^{*} M
$$

oú $\Lambda^{r, s} T_{\mathbb{C}}^{*} M=\Lambda^{r} \nu_{\mathbb{C}}^{*} \mathcal{F} \otimes \Lambda^{s} T_{\mathbb{C}}^{*} \mathcal{F}$ pour tout entier $i$.

Relativement à cette bigraduation, on constate que la différentielle $d$ s'écrit comme somme d' opérateurs $d_{1,0}, d_{0,1}$ et $d_{2,-1}$ de bidegrés respectifs $(1,0),(0,1)$ et $(2,-1)$. 
Rappelons qu'une forme différentielle $\omega \in \Omega^{i}(M):=\Gamma\left(\Lambda^{i} T_{\mathbb{C}} M\right)$ est dite basique si elle vérifie

$$
i_{X} \omega=i_{X} d \omega=0
$$

pour tout champ de vecteur $X$ tangent au feuilletage.Cette propriété est évidemment compatible avec le produit extérieur et la différentiation, de sorte que l'espace $\Omega^{*}(M / \mathcal{F})$ des formes basiques apparait comme un sous-complexe du complexe de de Rham usuel . Son homologie $H^{*}(M / \mathcal{F})$ est appelée cohomologie basique de $\mathcal{F}$.

Par la suite, on s'interessera aux cas des feuilletages transversalement holomorphe. Dans cette situation, $\mathcal{F}$ est défini par un cocycle feuilleté $\left\{U_{i}, f_{i}, \gamma_{i j}\right\}$, où $\left(U_{i}\right)$ est un recouvrement ouvert de $M, f_{i}: U_{i} \rightarrow S$ une submersion au dessus d'une variété holomorphe transverse $S$ de dimension complexe $n$ et $\gamma_{i j}$ un biholomorphisme local tel que sur $U_{i} \cap U_{j}$, on ait $f_{i}=\gamma_{i j} \circ f_{j}$.

Le fibré $\nu \mathcal{F}$ admet une structure complexe naturelle hérité de celle définie sur l'espace tangent de $M$. Cette structure induit, avec les notations usuelles, une décomposition en sous-espaces propres sur le complexifié de $\nu^{*} \mathcal{F}$

$$
\nu_{\mathbb{C}}^{*} \mathcal{F}=N^{*} \mathcal{F}^{1,0} \oplus N^{*} \mathcal{F}^{0,1}
$$

et détermine ainsi un scindage du fibré $\Lambda^{r} \nu_{\mathbb{C}}^{*} \mathcal{F}$ sous la forme

$$
\Lambda^{r} \nu_{\mathbb{C}}^{*} \mathcal{F}=\bigoplus_{p+q=r} N^{*} \mathcal{F}^{p, q}
$$

où $N^{*} \mathcal{F}^{p, q}=\Lambda^{p} N^{*} \mathcal{F}^{1,0} \otimes \Lambda^{q} N^{*} \mathcal{F}^{0,1}$.

Comme dans le cas ordinaire, $d_{1,0}$ se décompose comme somme de deux opérateurs $d_{1,0}=\partial+\bar{\partial}$ tels que

$$
\partial \Gamma\left(N^{*} \mathcal{F}^{p, q} \otimes \Lambda^{s} T_{\mathbb{C}}^{*} \mathcal{F}\right) \subset \Gamma\left(N \mathcal{F}^{* p+1, q} \otimes \Lambda^{s} T_{\mathbb{C}}^{*} \mathcal{F}\right)
$$

et

$$
\bar{\partial} \Gamma\left(N^{*} \mathcal{F}^{p, q} \otimes \Lambda^{s} T_{\mathbb{C}}^{*} \mathcal{F}\right) \subset \Gamma\left(N^{*} \mathcal{F}^{p, q+1} \otimes \Lambda^{s} T_{\mathbb{C}}^{*} \mathcal{F}\right) .
$$

Dans l'espace des formes basiques, la bigraduation précédente devient

$$
\Omega^{r}(M / \mathcal{F})=\bigoplus_{p+q=r} \Omega^{p, q}(M / \mathcal{F})
$$


avec $\Omega^{p, q}(M / \mathcal{F})=\Omega^{r}(M / \mathcal{F}) \cap \Gamma\left(N^{*} \mathcal{F}^{p, q}\right)$.

Soit $\mathcal{F}$ transversalement holomorphe; on dira que $\mathcal{F}$ est hermitien s'il existe sur $\nu \mathcal{F}$ une métrique hermitienne $g$ invariante par holonomie. Soit $n$ la codimension complexe de $\mathcal{F}$. Il est établi dans (voir [8]) qu'on a, lorsque la varité ambiante $M$ est compacte, l'alternative suivante :

a) $H^{2 n}(M / \mathcal{F})=\{0\}$

$\mathrm{Ou}$

b) $H^{2 n}(M / \mathcal{F})=\mathbb{C}$.

Un feuilletage admettant la propriété b) est dit homologiquement orientable. Dans ce cas, la classe (en cohomologie basique)de la forme volume transverse induite par $g$ est un générateur de $H^{2 n}(M / \mathcal{F})$

Soit $(\mathcal{F}, g)$ un feuilletage hermitien. Dans un système de coordonnées holomorphes $z=\left(z_{1}, \ldots, z_{n}\right)$ qui paramétre l'espace local des feuilles, la métrique transverse s'écrit

$$
g=\sum_{i, j} g_{i j}(z) d z_{i} d \overline{z_{j}}
$$

avec les conditions $g_{i j}=\overline{g_{j i}}$; Par analogie au cas usuel, on peut lui associer sa forme fondamentale $\eta$ et sa forme (oú courbure) de Ricci $\rho$. On a $\eta, \rho \in \Omega^{1,1}(M / \mathcal{F})$ et localement :

$$
\begin{gathered}
\eta=\sqrt{-1} / 2 \sum_{i, j} g_{i j} d z_{i} \wedge d \overline{z_{j}} \\
\rho=-\sqrt{-1} / \pi \partial \bar{\partial} \log \left(\eta^{n} /\left|d z_{1} \wedge \ldots \wedge d z_{n}\right|^{2}\right)
\end{gathered}
$$

Nous serons par la suite amené à considérer l'opérateur

$$
\bar{*}: \Omega^{*}(M / \mathcal{F}) \rightarrow \Omega^{2 n-*}(M / \mathcal{F})
$$

attaché á la métrique $g$ ainsi que les laplaciens basiques associés, $\delta=$ $d d^{\bar{*}}+d^{*} d$ et similairement $\Delta_{\partial}, \Delta_{\bar{\partial}}$ (pour une définition précise, le lecteur pourra par exemple consulter [6] ou [7]).

On focalisera notre attention sur les feuilletages kähleriens, c'est- á dire les feuilletages hermitiens tels que la forme fondamentale $\eta$ est 
fermée. Dans cette situation, on a, comme dans le cas classique les relations $\Delta=2 \Delta_{\partial}=2 \Delta_{\bar{\partial}}$.

Désignons par $\mathfrak{S}$ des germes de transformations infinitésimales de $\mathcal{F}$, par le sous-faisceau $\mathfrak{T}$ des germes champs de vecteurs tangents à $\mathcal{F}$ et par $\Xi:=\mathfrak{S} / \mathfrak{T}$ le faisceau des germes de champs basiques.

Soit $\mathcal{F}$ un feuilletage hermitien (ou plus généralement riemannien) sur $\mathrm{M}$ compacte. On note $\mathcal{C}$ son faisceau transverse central. Rappelons qu'il s'agit d'un sous-faisceau localement constant de $\Xi$ d'algèbres de Lie de dimension finie, introduit par Molino ([13]) et dont les sections locales sont des champs de Killing pour la métrique transverse $g$.

Les orbites de ces champs décrivent l'adhérence des feuilles de $\mathcal{F}$ au sens suivant : chaque point $m \in M$ admet un voisinage ouvert $V$ tel que la restriction $\mathcal{C}_{\mid V}$ soit un faisceau constant et tel que l'orbite de $m$ (plus exactement sa projection sur l'espace des feuilles local $V / \mathcal{F}$ ) soit ouverte dans(la projection de) l'adhérence de la feuille passant par $m$.

Ces germes de champs de Killing peuvent être caractérisés comme suit : les éléments du pseudo-groupe d'holonomie suffisamment proches de l'identité sont ceux de la forme $\exp \xi$ où $\xi$ est une section locale de $\mathcal{C}$; ce faisceau d'algèbre de Lie confère ainsi au pseudo-groupe d'holonomie une structure de pseudo-goupe de Lie (13], appendice d'E.Salem).

En particulier, lorsqu'on a affaire à un feuilletage hermitien, ces champs sont des parties réelles de champs holomorphes. En d'autres termes, si $X$ désigne un tel champ de Killing, on peut écrire $X-i J X$ sous la forme

$$
\sum a_{l}(z) \frac{\partial}{\partial z_{l}}
$$

dans un systéme de coordonnés transverses holomorphes $z=\left(z_{1}, \ldots, z_{n}\right)$, les $a_{l}$ étant holomorphes.

On peut facilement identifier le faisceau $\mathcal{C}$ sur l'exemple suivant. Soit $\left(M_{i}, g_{i}\right) i=1,2$ deux variétés hermitiennes complètes. Considérons alors la variété hermitienne $M_{1} \times M_{2}$ munie de la métrique produit et supposons qu'il existe un sous-goupe $\Gamma$ d'isométries holomorphes dont l'action est diagonale, libre, discrète et cocompacte. La variété quotient $M_{1} \times M_{2} / \Gamma$ est alors munie de deux feuilletages holomorphes en position transverse correspondant aux facteurs $M_{i} i=1,2$ (respectivement horizontal et vertical). Prenons par exemple le cas du feuilletage horizontal $\mathcal{F}$; l'action de $\Gamma$ étant diagonale, on peut étudier sa projection $\Gamma_{2}$ sur le second facteur $M_{2}$. Remarquons que $\Gamma_{2}$ n'est pas 
nécessairement fermé dans le groupe des isométries $\operatorname{Isom}\left(M_{2}\right)$ de $M_{2}$, en revanche, son adhérence est un groupe de Lie dont l'algèbre de Lie $\mathcal{C}_{0}$ est formée de champs de Killing (pour la métrique $g_{2}$ ). Le faisceau transverse central associé á $\mathcal{F}$ n'est alors rien d'autre que $\mathcal{C}=\pi_{*}\left(\mathcal{C}_{0}\right)$ oú $\pi: M_{1} \times M_{2} \rightarrow M_{1} \times M_{2} / \Gamma$ désigne l'application de revêtement.

Concluons cette section par une derniére définition.

Soit $\mathcal{F}$ un feuilletage transversalement holomorphe. Une $(1,1)$ forme réelle basique $\xi$ sera dite semi- négative si pour toute section $v$ de $N \mathcal{F}^{1,0}, \sqrt{-1} \xi(v \wedge \bar{v}) \leq 0$ et quasi-négative s'il existe de plus $v$ tel que $\sqrt{-1} \xi(v \wedge \bar{v})$ soit strictement négative en au moins un point de $M$. Nous retrouvons bien sûr la définition habituelle lorsque $\mathcal{F}$ est de dimension nulle!

\section{PrÉSEntation des RÉsultats et COMmentaires}

Sauf mention du contraire, $(\mathcal{F}, g)$ désignera dorénavant un feuilletage kählerien sur une variété $M$ compacte; $g$ est la métrique kahlerienne transverse et on note respectivement $\eta$ et $\rho$ sa forme fondamentale et sa forme de Ricci.

Theoréme 1.1-Supposons que $(\mathcal{F}, g)$ est homologiquement orientable et que de plus la courbure de Ricci $\rho$ est cohomologue (dans $\Omega^{2}(M / \mathcal{F})$ ) à une $(1,1)$ forme basique quasi-négative; alors le faisceau transverse central $\mathcal{C}$ est un faisceau en algèbres de Lie semi-simples.

Ce théorème est en fait un avatar feuilleté du résultat suivant obtenu par Nadel :

Theorème 1.2 ([14]) Soit $M$ une variété complexe compacte et connexe dont le fibré canonique est ample. Alors la composante neutre du groupe d'automorphismes Aut $(\tilde{M})$ ) de son revêtement universel $\tilde{M}$ est semi-simple.

Tel qu'il est articulé, cet énoncé sous-entend que $A u t(\tilde{M}))$ est muni d'une structure de groupe de Lie. C'est effectivement vrai et ceci résulte du fait qu'il agit par isométries sur $\tilde{M}$ pour la métrique Kähler Einstein relevée. Dans ce contexte, la courbure de Ricci est strictement négative et ceci précise les liens avec notre travail. Si $\left.A u t^{0}(\tilde{M})\right)$ est susceptible 
d'être riche, a contrario, les automorphismes de $M$ sont en nombre fini, phénomènes bien connus lorque $M$ est une surface de Riemann de genre supérieur ou égal à deux.

En fait, ces deux propriétés, á priori antagonistes sont toutes les deux des conséquences des formules de Weitzenböck-Bochner jointes à un lemme élémentaire mais fondamental de Nadel (loc.cit) concernant les algèbres de Lie abéliennes de champs de Killing et ces techniques s'adaptent parfaitement à la situation qui est la nôtre.

Signalons que l'utilisation nous faisons du principe de Bochner est très similaire à celle de Wu et Zheng dans [17] ( voir aussi [9]). Chez Nadel (loc.cit) cet argument est seulement mentionné et ce dernier fait plutôt usage de la stabilité du fibré tangent.

On obtient une formulation plus précise du théorème 1.1 en le spècialisant à la classe de feuilletages présentée ci-dessous.

Soit $M$ une variét'e kählerienne compacte munie d'un feuilletage holomorphe régulier $\mathcal{F}$. Notons respectivement $T_{\mathcal{F}}$ et $N_{\mathcal{F}}$ les fibrés tangent et normal de $\mathcal{F}$ munis de leur structure holomorphe usuelle. Supposons de plus que là classe canonique de $T \mathcal{F}$ est numériquement triviale,i.e :

$$
c_{1}\left(T_{\mathcal{F}}\right)=0
$$

Dans [16] est donnée une description assez précise de tels feuilletages en codimension 1. En codimension quelconque, nous obtenons ici le

Théorème 1.3 Soit $\mathcal{F}$ un feuilletage holomorphe régulier comme cidessus. On suppose en outre que $c_{1}\left(N_{\mathcal{F}}\right)$ est représentée par une $(1,1)$ forme fermée semi-négative et vérifie $c_{1}(M)^{n} \neq 0$; alors, á revêtement fini prés, $\mathcal{F}$ est une fibration localement triviale au dessus d'une variété $V$ à première classe de Chern quasi-négative (en particulier, les feuilles de $\mathcal{F}$ sont fermées).

Corollaire 1.1 Sous les hypothèses du théorème 1.2, $\mathcal{F}$ coïncide avec la fibration de d'Itaka-Kodaira de $M$.

Théorème 1.4 Soit $\mathcal{F}$ un feuilletage holomorphe régulier de codimension $n \leq 2$ à classe canonique numériquement triviale sur $M$ kähler compacte. On suppose en outre que $c_{1}\left(N_{\mathcal{F}}\right)$ est représentée par une $(1,1)$ forme fermée semi-négative; alors il existe un feuilletage holomorphe $\mathcal{G}$ de contenant $\mathcal{F}$ et tel que $c_{1}(N \mathcal{G})=c_{1}(N \mathcal{F})$ et les feuilles 
de $\mathcal{G}$ soient fermées.

Nous conjecturons que cet énoncé reste valide en laissant tomber l'hypothèse sur la codimension. Comme nous le rappelons en section 4 , c'est effectivement le cas quand $\mathcal{F}$ est défini par l'action localement libre d'un groupe de Lie complexe et ceci résulte simplement des travaux de Lieberman ([1]]) relatifs au groupes d'automorphismes des variétés kähleriennes.

En dehors des ingrédients déjà mentionnés, la démonstration du théorème 1.1 passe par l'obtention d'un lemme $d d^{c}$ pour les courants basiques. C'est l'objet de la section qui suit.

\section{Courants Basiques}

Soit $\mathcal{F}$ un feuilletage de dimension $n$ sur une variété $M$ orientée de dimension (réelle) $N$.

On notera $\Gamma \mathcal{F}$ le $\mathcal{C}^{\infty}(M)$ module des champs de vecteurs sur $M$ tangent à $\mathcal{F}$ et on désignera par $\Omega_{c}^{*}(M)$ (oú simplement $\Omega^{*}(M)$ quand $M$ est compacte) le complexe des formes différentielles sur $M$ à valeurs complexes et à support compact.

Un courant $T \in \Omega_{\mathbb{C}}^{*}(M)^{\prime}$ de degré $r$ est dit basique si, pour tout $X \in \Gamma \mathcal{F}, i_{X} T=0$ et $L_{X} T=0$, le produit intérieur $i_{X}$ et la dérivée de Lie $L_{X}$ d'un $r$ courant $T$ étant définis par dualité comme suit:

Pour tout champ de vecteurs $X$ sur $M$ et pour toute forme $\alpha \in$ $\Omega_{c}^{N-r}(M)^{\prime}$,

$$
\begin{aligned}
\left\langle i_{X} T, \alpha\right\rangle & =(-1)^{r}\left\langle T, i_{X} \alpha\right\rangle \\
\left\langle L_{X} T, \alpha\right\rangle & =(-1)^{r}\left\langle T, L_{X} \alpha\right\rangle .
\end{aligned}
$$

On notera $\mathcal{C}_{\mathcal{F}}^{*}(M)$ le complexe des courants basiques sur $M$.

Une $r$ forme $\eta$ définit un courant $T_{\eta}$ de degré $r$, dit régulier par la formule habituelle

$$
\left\langle T_{\eta}, \alpha\right\rangle=\int_{M} \eta \wedge \alpha
$$

où $\alpha$ est une $N-r$ forme á support compact.

On peut vérifier ([回]) que $T_{\eta}$ est basique si et seulement si $\eta$ est basique. 
Quand $\mathcal{F}$ est transversalement holomorphe de codimension complexe $r=\frac{N-n}{2}$, un courant basique sera dit par ailleurs de bidegré $(p, q)$ s'il appartient au dual topologique de $\Gamma\left(N^{*} \mathcal{F}^{r-p, r-q} \otimes \Lambda^{n} T_{\mathbb{C}}^{*} \mathcal{F}\right) \cap \Omega_{c}^{*}(M)$. On note $\mathcal{C}_{\mathcal{F}}^{p, q}(M)$ l'ensemble de tels courants. On obtient ainsi une décomposition des courants basiques de degré $r$,

$$
\mathcal{C}_{\mathcal{F}}^{r}(M)=\bigoplus_{p+q=r} \mathcal{C}_{\mathcal{F}}^{p, q}(M)
$$

et un double complexe

$$
\left(\mathcal{C}_{\mathcal{F}}^{*, *}(M), \partial, \bar{\partial}\right)
$$

Les opérateurs $\partial$ et $\bar{\partial}$ s'étendent aux courants basiques comme suit :

si $T \in \mathcal{C}_{\mathcal{F}}^{r}(M)$, on pose pour tout $\omega \in \Omega_{c}^{N-r}(M)$

$$
\langle\partial T, \omega\rangle=(-1)^{r+1}\langle T, \partial \omega\rangle
$$

et

$$
\langle\bar{\partial} T, \omega\rangle=(-1)^{r+1}\langle T, \bar{\partial} \omega\rangle
$$

Le cadre considéré à partir de maintenant est celui d'une variété compacte $M$ munie d'un feuilletage kählérien de dimension $p$ et de codimension complexe $n$. nous supposerons en outre que $\mathcal{F}$ est homologiquement orientable. Cette dernière condition implique, suivant un résultat de Xosé Masa ([12]), que $\mathcal{F}$ est minimalisable. Ceci revient à dire qu'il existe sur $M$ une métrique riemannienne $\tilde{g}$ dont le volume sur les feuilles est la restriction d'une forme $\chi \in \Omega^{p}(M)$ relativement fermée :

pour tous champs de vecteurs $X_{1}, \ldots, X_{p+1}$ tels que $X_{i} \in \Gamma(T \mathcal{F}), i=$ $1, \ldots, p$, on a

$$
d \chi\left(X_{1}, \ldots, X_{p+1}\right)=0 .
$$

On choisira par la suite $\tilde{g}$ de telle sorte que la métrique induite sur $\nu \mathcal{F}$ soit précisément $g$.

L'opérateur $₹$ est alors relié á l'opérateur * ordinaire (associé à $\tilde{g}$ ) par la formule

$$
* u=\bar{*} u \wedge \chi
$$

Pour toute forme $\alpha, \beta \in \Omega^{r}(M / \mathcal{F})$, on obtient donc que 


$$
(\alpha, \beta)=\int_{M} \alpha \wedge \bar{*} \beta \wedge \chi
$$

où pour tout $\xi, \eta \in \Omega^{s}(M),(\xi, \eta)=\int_{M} \xi \wedge * \eta$ est le produit scalaire hermitien induit par $\tilde{g}$ sur $\Omega^{*}(M)$.

Compte-tenu de la relation (2.1), on a par ailleurs que pour tout $u \in \Omega^{r}(M / \mathcal{F})$ et pour tout $\xi \in \Omega^{N-r}(M)$,

$$
\left\langle T_{\bar{*} u}, \xi\right\rangle=\int_{M} \bar{*} \wedge \wedge \xi= \pm \int_{M} *(u \wedge \chi) \wedge \xi= \pm \overline{\left\langle T_{u}, * \xi \wedge \chi\right\rangle},
$$

ce qui permet d'expliciter l'extension de $₹$ (et par suite de $\Delta$ ) á l'espace des courants basiques.

On peut montrer qu'on obtient, relativement au produit scalaire précédent, une décomposition de Hodge basique pour l'opérateur auto-adjoint $\Delta$ (voir [6], [7]), i.e., une décomposition en sous-espace orthogonaux du type

$$
\Omega^{p, q}(M / \mathcal{F})=\mathcal{H}^{p, q} \oplus \operatorname{Im} \Delta
$$

où $\mathcal{H}^{p, q}=\operatorname{ker} \Delta$ est de dimension finie (par abus de langage, on note encore $\Delta$ la restriction du laplacien basique á $\left.\Omega^{p, q}(M / \mathcal{F})\right)$.

Soit $\omega \in \Omega^{p, q}(M / \mathcal{F})$ et $H(\omega)$ sa projection orthogonale sur $\mathcal{H}^{p, q}$; considérons l'opérateur de Green $G: \Omega^{p, q}(M / \mathcal{F}) \rightarrow \Omega^{p, q}(M / \mathcal{F})$ (on rappelle que $G \omega$ est l'unique forme telle que $G \omega \in \operatorname{Im} \Delta$ et $\Delta G \omega=$ $\omega-H(\omega))$.

Comme dans le cas classique,

$\omega=H(\omega)+\Delta G=H(\omega)+\partial \partial^{\bar{*}} G \omega+\partial^{\bar{*}} \partial G \omega=H(\omega)+\bar{\partial} \bar{\partial}^{\bar{*}} G \omega+\bar{\partial}^{\bar{*}} \bar{\partial} G \omega$

et $G$ commute aux différents opérateurs $d, \partial, \bar{\partial}, \bar{*}$.

Dans ce qui suit, on veut établir qu'il existe une décomposition similaire pour $T \in \mathcal{C}_{\mathcal{F}}^{p, q}(M)$. Pour ce faire, on va utiliser qu'il existe une suite de formes basiques $\left(u_{n}\right)$ de bidegré $(p, q)$ telle que la suite de courant basiques $\left(T_{u_{n}}\right)$ converge vers $T$ ([四]). En effet, soit $\left(u_{n}\right)$ une telle suite; pour tout entier $n$, on a

$$
T_{u_{n}}=T_{H\left(u_{n}\right)}+T_{\Delta G u_{n}}
$$


Puisque $\mathcal{H}^{p, q}$ est de dimension finie, il est clair que $\left(T_{H\left(u_{n}\right)}\right)$ converge vers $T_{H(T)}$ où $H(T) \in=\mathcal{H}^{p, q}$ est l'unique forme harmonique telle que pour tout $h \in \mathcal{H}^{n-p, n-q}$, on ait

$$
\int_{M} H(T) \wedge h \wedge \chi=\langle T, h \wedge \chi\rangle
$$

Par suite, $\left(T_{\Delta G_{u_{n}}}=\Delta T_{G_{u_{n}}}\right)$ converge vers un courant basique $T^{\prime}$. Nous affirmons que la suite de courants $\left(T_{G_{u_{n}}}\right)$ est convergente, auquel cas $T^{\prime}=\Delta T^{\prime \prime}$ avec $T^{\prime \prime}=\lim _{n \rightarrow+\infty} T_{G_{u_{n}}} \in \mathcal{C}_{\mathcal{F}}^{p, q}(M)$. Ceci se prouve aisément en invoquant le résultat suivant :

Théorème 2.1 ([10]) Il existe un opérateur elliptique

$$
D: \Omega^{*}(M) \rightarrow \Omega^{*}(M)
$$

préservant le degré (non nécessairement auto-adjoint) tel que $D$ coïncide avec $\Delta$ en restriction à $\Omega^{*}(M / \mathcal{F})$ et tel que la décomposition orthogonale (donnée par la théorie classique)

$$
\Omega^{*}(M)=\operatorname{ker} D \oplus \operatorname{Im} D^{*}
$$

soit compatible avec celle de $\Omega^{*}(M / \mathcal{F})$; en d'autre termes, ker $\Delta \subset$ ker $D$ et $\operatorname{Im} \Delta \subset \operatorname{Im} D^{*}$.

Etabissons la convergence de $T_{G_{u_{n}}}$; pour tout $v \in \Omega^{p+q}(M)$, on a

$$
\left\langle T_{\Delta G u_{n}}, * v\right\rangle=\left\langle T_{G u_{n}}, * D^{*} v\right\rangle .
$$

Par ailleurs, pour tout $h \in \operatorname{Ker} D$, on a

$$
\left\langle T_{G u_{n}}, * h\right\rangle=\left(T_{G u_{n}}, h\right)=0,
$$

en conséquence de quoi $\left(T_{G u_{n}}\right)$ converge (faiblement) vers le courant $T^{\prime \prime}$ défini par

$$
\begin{aligned}
& \left\langle T^{\prime \prime}, * D^{*} * v\right\rangle=\lim _{n \rightarrow+\infty}\left\langle T_{\Delta G u_{n}}, * v\right\rangle \text {, pour tout } v \in \Omega^{p+q}(M) \text { et } \\
& \left\langle T^{\prime \prime}, * h\right\rangle=0 \text {, pour tout } h \in \operatorname{Ker} D \cap \Omega^{p+q}(M) .
\end{aligned}
$$

La série d'observations précédentes permet alors d'énoncer la

Proposition 2.1 Soit $(\mathcal{F}, g)$ un feuilletage kahlerien sur $M$ compacte. Soit $T$ un courant basique de bidegré $(p, q)$; alors $\Delta T=0$ si et seulement si $T=T_{h}$ où $h \in \mathcal{H}^{p, q}$ et on hérite d'une décomposition en somme directe 


$$
\mathcal{C}_{\mathcal{F}}^{p, q}(M)=\operatorname{ker} \Delta \oplus \operatorname{Im} \Delta .
$$

L'opérateur de Green $G: \mathcal{C}_{\mathcal{F}}^{p, q}(M) \rightarrow \mathcal{C}_{\mathcal{F}}^{p, q}(M)$ associé á cette décomposition (défini similairement au précédent) commute à $d, \partial, \bar{\partial}, \bar{*}$.

De plus, si $T$ est $d$ exact (i.e, il existe $T^{\prime} \in \mathcal{C}_{\mathcal{F}}^{p+q-1}(M)$ tel que $d T^{\prime}=$ $T)$, on a alors

$$
T=2 \partial \partial^{\bar{*}} G T=2 \bar{\partial} \bar{\partial}^{\bar{*}} G T .
$$

Corollaire 2.1 Soit $T \in \mathcal{C}_{\mathcal{F}}^{p, q}(M) d$ exact; alors $T$ est $\partial \bar{\partial}$ exact; c'est-à-dire qu'il existe un courant $T^{\prime} \in \mathcal{C}_{\mathcal{F}}^{p-1, q-1}(M)$ tel que

$$
T=\sqrt{-1} \partial \bar{\partial} T^{\prime} .
$$

De plus, on peut choisir $T^{\prime}$ réel lorsque $T \in \mathcal{C}_{\mathcal{F}}^{p, p}(M)$ est réel.

\section{Preuve DU théorème 1.1}

On rappelle que, par hypothèse, $(\mathcal{F}, g)$ désigne un feuilletage transversalement kahlerien et homologiquement orientable dont la courbure de Ricci transverse $\rho$ est cohomologue $\left(\right.$ dans $\Omega^{*}(M / \mathcal{F})$ ) à une $(1,1)$ forme réelle basique quasi-négative $\kappa$. Quitte à se placer sur un double revêtement, on peut également supposer que $\mathcal{F}$ est orientable.

Après résolution de l'équation de Monge-Ampère basique ([6]), on se ramène au cas où $\kappa=\rho$. Un des points clés de la démonstration est certainement le lemme suivant qui est de nature purement locale :

Lemme 3.1. ( 14 )- Soient $U$ un ouvert connexe de $\mathbb{C}^{n}$ muni d'une métrique kahlerienne $g$ (non nécessairement complète) et $X_{1}, \ldots, X_{l}$ des champs de vecteurs holomorphes sur $U$ commutant 2 à 2 tels que leur partie réelle $\operatorname{Re}\left(X_{i}\right), i=1, \ldots, l$ soit un champ de Killing.

On suppose de plus que les $X_{i}, i=1, \ldots, n$ forment une famille libre sur $\mathbb{R}$ mais sont méromorphiquement dépendants; alors la courbure de Ricci $\rho$ n'a de signe défini en aucun point de $U$.

Supposons par l'absurde que $\mathcal{C}$ ne soit pas un faisceau d'algèbre semi-simples. Chaque germe d'algèbre $\mathcal{C}_{m}, m \in M$ admet donc un radical rèsoluble $R, m$ non trivial, lequel est unique. La collection des 
$R_{m}$ dèfinit donc un faisceau localement d'algèbre de Lie rèsolubles. Similairement, on peut considérer le sous-faisceau localement constant $\mathcal{A}$ dont la fibre, en chaque point $m$, est la dernière algèbre dérivée non triviale.

Par "complexification", on hérite alors d'un fasceau localement constant $\mathcal{A}_{\mathbb{C}}$ d'algèbres de Lie de champs de vecteurs holomorphes. Plus précisément, $\mathcal{A}_{\mathbb{C}}$ est engendrée sur $\mathbb{C}$ à partir des sections locales $X-i J X$ où $X$ est une section locale de $\mathcal{A}$ et $J$ la structure complexe transverse. Remarquons que $d:=\operatorname{Dim}_{\mathbb{C}} \mathcal{A}_{\mathbb{C}} \leq \operatorname{Dim}_{\mathbb{R}} \mathcal{A}$.

Par évaluation des champs de vecteurs, on récupère un morphisme de faisceaux :

$$
e v: \mathcal{A}_{\mathbb{C}} \rightarrow N_{\mathcal{F}}^{1,0}
$$

qui induit un morphisme :

$$
\bigwedge^{d} e v: \operatorname{det} \mathcal{A}_{\mathbb{C}}=\bigwedge^{d} \mathcal{A}_{\mathbb{C}} \rightarrow \bigwedge^{d} N_{\mathcal{F}}^{1,0}
$$

Compte-tenu du lemme 3.1 et de l'hypothèse $\rho$ quasi-négatif", ce morphisme n'est pas trivial et on récupère par ce procédé une section holomorphe non nulle $s$ du fibré $E:=\bigwedge^{d} N_{\mathcal{F}}^{1,0} \otimes\left(\operatorname{det} \mathcal{A}_{\mathbb{C}}\right)^{-1}$.

On peut donc trouver un recouvrement ouvert $\mathcal{U}=\left\{U_{i}\right\}$ de $M$ de telle sorte qu'en restriction á chaque $U_{i}, s$ soit représenté par une section holomorphe $s_{i}$ de $\bigwedge^{d} N_{\mathcal{F}}^{1,0}$ telle que sur chaque intersection $U_{i} \cap U_{j}$,

$$
s_{i}=g_{i j} s_{j}
$$

où $\left(g_{i j}\right)$ est un cocycle multiplicatif localement constant.

Soit $g_{d}$ la métrique induite par $g$ sur le fibré $\bigwedge^{d} N_{\mathcal{F}}^{1,0}$. Sur $U_{i} \cap U_{j}$, on a

$$
g_{d}\left(s_{i}, \overline{s_{i}}\right)=\left|g_{i j}\right|^{2} g_{d}\left(s_{j}, \overline{s_{j}}\right) .
$$

Par ailleurs,il est clair que pour tout indice $i, \varphi_{i}=\log \left(g_{d}\left(s_{i}, \overline{s_{i}}\right) \in\right.$ $L_{l o c}^{1}\left(U_{i}\right)$ et ces fonction sont localement constantes sur les feuilles de $\mathcal{F}_{\mid U_{i}}$. Par recollement, la collection des $\sqrt{-1} \partial \bar{\partial} \varphi_{i}=$ (bien définis au sens de courants) produit un courant basique $T$ de bidegré $(1,1) d$ exact (en cohomologie basique). Il résulte alors du corollaire 2.1 que

$$
T=\sqrt{-1} \partial \bar{\partial} u
$$


où $u \in \mathcal{C}_{\mathcal{F}}^{0}(M)$ est une distribution basique réelle. Sur tout ouvert $U_{i}$, on obtient ainsi que

$$
u-T_{\varphi_{i}}=T_{\log \left|H_{i}\right|^{2}}
$$

où $H_{i}$ est une fonction holomorphe basique (pour le feuilletage restreint) ne s'annulant pas sur $U_{i}$. Il en résulte que pour tout $i$,

$$
H_{i} s_{i}=h_{i j} H_{j} s_{j}
$$

où les $h_{i j}$ sont localement constants de module 1 .

Compte-tenu de ce qui précède, les $\left\|H_{i} s_{i}\right\|^{2}$ se recollent sur les intersections en une fonction positive $\xi$ telle que, suivant la formule de Weintzenböck, on ait

$$
\triangle \xi \supsetneqq, 0
$$

ce qui contredit le théorème de Hopf.

Remarque 3.1 : Les mêmes démonstrations et la conclusion du théorème 1.1 conclusions restent valides en remplaçant $\mathcal{C}$ par n'importe quel faisceau localement constant de champs de killing transverses qui sont des parties réelles de champs basiques holomorphes locaux.

\section{Feuilletages À Classe CANonique numériquement TRIVIALE}

Dans cette section, on désignera par $\mathcal{F}$ un feuilletage holomorphe régulier sur une variété $(M, g)$ kähleriene compacte de dimension complexe $m$ qui satisfait une partie des hypothéses du théorème 1.1, á savoir $\mathcal{F}$ est à classe canonique numériquement triviale, i.e, $c_{1}\left(T_{\mathcal{F}}\right)=0$; on supposera en revanche la classe anticanonique $c_{1}(M)$ représentée par une $(1,1)$ forme fermée $\eta$ seulement semi-négative (et à priori non basique).

\subsection{Preuve du théorème 1.3}

Elle résulte de la série d'observations suivantes.

Lemme 4.1 Il existe sur $M$ une métrique kahlerienne pour laquelle le feuilletage $\mathcal{F}$ (plus exactement le fibré $T_{\mathcal{F}}$ ) est parallèle. En particulier, 
$\eta$ est une forme basique de $\mathcal{F}$.

Preuve-. Suivant le fameux théorème de Yau (18 ), on peut munir $M$ d'une métrique kählerienne dont la courbure de Ricci est précisément $\eta$. Par hypothèse, il existe une section holomorphe non triviale du fibré en droites $\bigwedge^{m} T_{\mathcal{F}} \otimes E$ où $E$ est un certain fibré plat. Le lemme résulte alors du principe de Bochner tel qu'il est par exemple utlilisé dans la section précédente (voir aussi [16]).

Notons dorénavant par $g$ la métrique ainsi produite. On hérite par parallélisme d'une décomposition orthogonale et holomorphe du fibré tangent de $M$ :

$$
T M=T_{\mathcal{F}} \oplus T_{\mathcal{F}}{ }^{\perp}
$$

où $T_{\mathcal{F}}{ }^{\perp}=T_{\mathcal{G}}$ est le fibré tangent d'un feuilletage holomorphe $\mathcal{G}$ également parallèle.

Ce scindage, de nature infinitésimale, donne lieu par le théorème de de Rham á une décomposition du revêtement universel $\tilde{M}$ en produit kahlerien pour la métrique relevée $h=h_{1} \oplus h_{2}$ :

$$
\tilde{M}=\left(V, h_{1}\right) \times\left(V^{\perp}, h_{2}\right) .
$$

Par construction, la métrique $h_{1}$ est Ricci plate, ce qui va permettre de préciser la structure de $V$.

Lemme 4.2 La variété $V$ se scinde (holomorphiquement et isométriquement) sous la forme $\mathbb{C}^{k} \times N$ où $N$ est une variété de Calabi-Yau.

La démonstration de ce lemme suit celle qui est présentée dans [16]; nous la reproduisons par commodité pour le lecteur.

Elle repose notamment sur le résultat suivant de Cheeger et Gromoll.

\section{Théorème 4.1 (四)}

Soit $M$ une variété lisse simplement connexe admettant une métrique riemannienne complète de courbure de Ricci positive ou nulle; alors $M$ se décompose isométriquement sous la forme $\mathbb{R}^{k} \times N$ où $N$ est une variété ne contenant pas de droite géodésique.

Rappelons qu'une droite géodésique est une géodésique

$$
\gamma:]-\infty,+\infty[\rightarrow M
$$


telle qu'à chaque instant $t, t^{\prime} \in \mathbb{R}$, le segment $\gamma_{\mid\left[t, t^{\prime}\right]}$ soit minimal.

Preuve du lemme 4.2-. Puisque la décomposition de De Rham d'une variété kählerienne coïncide avec celle de la variété réelle sous-jascente, on obtient que $V$ se scinde holomorphiquement et isométriquement sous la forme $\mathbb{C}^{l} \times N$.

Par construction, le groupe fondamental de $M$ agit diagonalement par isométries sur le produit $\tilde{M}=\mathbb{C}^{l} \times N \times V^{\perp}$. Il reste à prouver que $N$ est compacte; supposons par l'absurde que ce ne soit pas le cas. La contradiction recherchée résulte alors de l'argument suivant repris verbatim à Cheeger et Gromoll (loc.cit). Soit $K$ un domaine fondamental pour l'action de $\pi_{1}(M)$ sur $\tilde{M}$. C'est un compact et sa projection $\rho(K)$ sur $N$ est donc un compact dont l'orbite par $\rho\left(\pi_{1}(M)\right)$ est $N$ toute entière. Puisque $N$ est supposée non compacte, il existe en un point $p \in \rho(K)$ un rayon géodésique $\gamma:[0, \infty[\rightarrow V$ (i.e une demi-droite géodésique) tel que $\gamma(0)=p \in \rho(K)$. Soit $g_{n}$ une suite d'isométries de $\rho\left(\pi_{1}(M)\right)$ telle que $g_{n}(\gamma(n))=p_{n} \in \rho(K)$. La géodésique $\gamma_{n}$ de $N$ définie par $\gamma_{n}(0)=p_{n}$ et $\gamma_{n}^{\prime}(0)=d g_{n}\left(\gamma^{\prime}(n)\right)$ est donc un rayon géodésique en restriction à $[-n, \infty[$. Par compacité, on peut extraire une sous-suite $n_{i}$ de sorte que $p_{n_{i}}$ et $\gamma_{n_{i}}{ }^{\prime}(0)$ convergent respectivement vers $p_{0} \in \rho(K)$ et $v_{0} \in T_{p_{0}}^{1}(N)$. Par suite, la géodésique passant en $p_{0}$ à la vitesse $v$ est une droite géodésique.

Lemme 4.3 Supposons de plus que les feuilles de $\mathcal{F}$ sont fermées, alors il existe un revêtement holomorphe fini de $M$ tel que le feuilletage pull-back soit une fibration holomorphe localement triviale.

Preuve du lemme 4.3-. Le groupe fondamental $\pi_{1}(M)$ agit sur $\tilde{M}=$ $\mathbb{C}^{k} \times N \times V^{\perp}$. diagonalement par isométries biholomorphes. Quitte à substituer à $M$ un revêtement fini, on peut supposer que $\pi_{1}(M)$ agit trivialement sur le facteur Calabi-Yau $N$ (car son groupe d'isométries est fini ([3])). Le lemme 4.3 sera établi si l'on arrive à extraire de $\pi_{1}(M)$ un sous groupe d'indice fini dont l'image par la projection $\rho: \pi_{1}(M) \rightarrow V \perp$ est sans torsion. Cela s'effectue en suivant pas à pas le raisonnement mené dans [17], p.279. le

D'aprés ce qui précède, le théorème 1.3 sera démontré une fois acquis

Lemme 4.4 Supposons que $c_{1}(M)$ soit représentée par une forme quasi-négative; alors l'action de $\pi_{1}(M)$ sur $V^{\perp}$ est discrète. 
Puisque le groupe des isométries de $N$ est fini ([3]), on est ramené à considérer un groupe $H$ dont l'action est libre, discrète, diagonale, cocompacte et isométrique sur le produit $\mathbb{C}^{l} \times V^{\perp}$. Il reste à voir que cette action reste discrète sur le second facteur, auquel cas chaque feuille de $\mathcal{F}$ sera fermée, ce qui achèvera la preuve du théoréme 1.3.

Preuve du lemme 4.4-. Puisque l'action du groupe fondamental est diagonale, on récupère un morphisme $\rho$ de $\pi_{1}(M)$ vers le groupe de Lie $\operatorname{Isom}\left(V^{\perp}\right)$ des isométries holomorphes de $V^{\perp}$. Soit $G$ la composante neutre de l'adhérence de $\rho\left(\pi_{1}(M)\right.$ dans $\operatorname{Isom}\left(V^{\perp}\right)$ et $\mathfrak{g}$ son algèbre de lie. Suivant un résultat dû à Eberlein, (汭), $\mathfrak{g}$ est résoluble; elle est par ailleurs semi-simple compte tenu du théorème 1.1f.Par suite, $\mathfrak{g}=\{0\}$ et le lemme est démontré.

Preuve du corollaire 1.1-. On peut supposer (lemme 4.3) que $\mathcal{F}$ est une fibration localement triviale. D'après [15], la base $V$ de cette fibration est de type général D'autre part, les fibres sont à première classe Chern nulle et ceci implique qu'il exite un entier $n$ non nul tel que $K_{\mathcal{F}}^{\otimes n}$ soit trivial. Le corollaire résulte alors simplement du fait que $K_{M^{\prime}}=\pi^{*} K_{V} \otimes K_{\mathcal{F}}$.

\subsection{Preuve du théorème 1.4}

Examinons d'abord le cas où le feuilletage en question est donnée par une action localement libre d'un groupe de Lie complexe $G$; le théorème 1.4 est une conséquence directe, sans hypothèse sur la codimension, d'un résultat démontré idépendamment par Lieberman et Fujiki. Ce résultat stipule que la composante connexe $A u t_{0}(M)$ du groupe des automorphismes d'une variété kählerienne compacte $M$ jouit des mêmes propriétés qu'un groupe algébrique; en particulier, le feuilletage holomorphe $\mathcal{G}$ (peut- être singulier) d éfini par les orbites de $A u t_{0}(M)$ (où celles de l'adhérence de Zariski de $G$ ) est à feuilles fermées (dans le complémentaire du lieu singulier éventuel). En courbure de Ricci semi-négative, tout champ de vecteur holomorphe est de plus parallèle d'après le principe de Bochner. Ceci nous assure que $\mathcal{G}$ est défini par une action localement libre et qu'en particulier $c_{1}(T \mathcal{G})=0$.

\footnotetext{
${ }^{1}$ On peut le déduire plus simplement du principe de Bochner usuel (cf.[17]) mais il nous a semblé interessant d'établir un résultat plus général
} 
Le théorème 1.4 a déjà été établi lorsque $c_{1}(M)$ est de rang maximal $n=\operatorname{codim} \mathcal{F}$ quelque part $(\mathcal{G}=\mathcal{F}$ convient) et le seul cas consistant à traiter est évidemment $n=2$.

Soit $G$ la composante neutre de $\overline{\rho\left(\pi_{1}(M)\right.}$ dont on rappelle que l'algèbre de Lie $\mathfrak{g}$ est résoluble. Les conclusions du théorème sont bien sur vérifiées si celle-ci est triviale. On supposera donc que ce n'est pas le cas.

Désignons par $\mathfrak{h}$ sa dernière algèbre de Lie dérivée non triviale; elle est globalement $\pi_{1}(M$ invariante et admet pour base $p$ champs de Killing $v_{1}, \ldots, v_{p}$, chaque $v_{i}$ étant la partie réelle d'un champ de vecteur holomorphe $X_{i}$ sur $V^{\perp}$. Par définition de $\mathfrak{h}$, les champs $V_{i}$, et par suite les champs $X_{i}$ commutent deux à deux. Notons $p^{\prime}$ la dimension complexe de l'algèbre de Lie $\mathfrak{h}_{\mathbb{C}}$ engendrée sur $\mathbb{C}$ par les $X_{i}$. Il est clair que $p^{\prime} \leq p$.

Supposons d'abord que $X_{1} \wedge X_{2} \neq 0$. On en déduit aisément, par abélianité, que $\left\{X_{1}, X_{2}\right\}$ forme une base de $\mathfrak{h}_{\mathbb{C}}$.

On a dautre part une représentation

$$
\pi_{1}(M) \rightarrow G L\left(\mathfrak{h}_{\mathbb{C}}\right)
$$

qui donne donc lieu à un fibré holomorphe plat de rang 2 sur la variété $M$. On récupère par ce procédé une section non triviale $s$ du fibré $\bigwedge^{n} T M \otimes \bigwedge^{2} E^{-1}$. Par les techniques de Bochner déjà mentionnées, $s$ est parallèle et ne s'annule donc pas; en conséquence de quoi on a $c_{1}(M)=0$ et le théorème 1.4 est montré ( $\mathcal{G}$ est ici de codimension nulle!).

Similairement, on peut construire sous l'hypothèse $p^{\prime}=1$ un feuilletage $\mathcal{G}$ de codimension 1 satisfaisant les conclusions du théorème 1.4..

Il reste donc à considérer le cas $p \geq 2$ avec $X_{i} \wedge X_{j}=0$ pour tout couple d'indices $(i, j)$. Quitte à renuméroter les indices, on peut supposer que $X_{1}, \ldots, X_{p^{\prime}}$ forment une base de $\mathfrak{h}_{\mathbb{C}}$.

Soit $m \in V^{\perp}$ tel que $X_{1}(m) \neq 0$ et $\left(z_{1}, z_{2}\right)$ un système de coordonnées holomorphes locales en $m$

On a alors

$$
X_{1}=\frac{\partial}{\partial z_{1}}
$$

$$
X_{i}=a_{i}\left(z_{2}\right), \frac{\partial}{\partial z_{1}} i=2, \ldots, p^{\prime}
$$

$a_{i}$ holomorphes non constantes.

En exploitant le fait que les $v_{k}, k=1, \ldots, p^{\prime}$ sont de Killing pour la métrique kälherienne 


$$
g^{\perp}=\sum_{i, j=1,2} g_{i j} d z_{i} \otimes d \overline{z_{j}}
$$

on obtient facilement que $\left(a_{k}-\overline{a_{k}}\right) g_{11}$ est constant pour tout $k$. Il en résulte que $p^{\prime}=2$ et que le champ de bivecteur $R e X_{1} \wedge \operatorname{Re} X_{2}$ est de norme constante non nulle. Par suite, $R e X_{1}$ et $R e X_{2}$ sont les générateurs d'un feuilletage holomorphe $\tilde{\mathcal{G}}$ sur $V^{\perp}$ défini localement par l'équation $\left\{d z_{2}=0\right\}$. Notons que $\tilde{\mathcal{G}}$ est hermitien, car déterminé par l'action de champs de Killing (la métrique transverse invariante est celle induite sur $T \tilde{\mathcal{G}}^{\perp}$ par $h_{2}$ ). Ce feuilletage est invariant sous l'action de $\overline{\rho\left(\pi_{1}(M)\right.}$, vu que $\mathfrak{h}$ l'est. De ces quelques remarques, il résulte que le feuilletage $\rho^{*}(\tilde{\mathcal{G}})$ se redescend sur $M$ en un feuilletage $\mathcal{G}$ holomorphe de codimension 1 complexe hermitien (et donc kählerien).

Lemme 4.5 Les feuilles de $\mathcal{G}$ sont fermées

Preuve-. Supposons par l'absurde que ce n'est pas le cas. Soit $\left\{X_{1}, X_{2}=\right.$ $\left.f X_{1}\right\}$ une base de $\mathfrak{h}_{\mathbb{C}}$ D'aprés ce qui précéde, on a $X_{2}==f X_{1}$ oú $f$ est une fonction holomorphe dont la partie imaginaire ne s'annule pas et telle que $X_{1}(f)=0$. Pour tout $g \in \rho\left(\pi_{1}(M)\right)$, il existe manifestement une matrice

$$
\left(\begin{array}{ll}
a & b \\
c & d
\end{array}\right) \in G l_{2}(\mathbb{R})
$$

telle que

$$
\left\{\begin{array}{l}
g_{*} X_{1}=a X_{1}+b X_{2} \\
g_{*} X_{2}=c X_{1}+d X_{2}
\end{array}\right.
$$

Par suite, la $(1,1)$ forme $\sqrt{-1} / 2 \pi \frac{d f \wedge \bar{f}}{|f-\bar{f}|^{2}}$ se redescend sur $M$ en une métrique (éventuellement dégénérée) transverse á $\mathcal{G}$ et invariante par holonomie définie par une $(1,1)$ forme $\eta$. Le lieu des zéros de $\eta$ est une union finie d'hypersurfaces $\bigcup_{i=1}^{l} H_{i}$ invariantes par $\mathcal{G}$. Cette forme $\eta$ permet de munir le fibré normal $N_{\mathcal{G}}$ d'une métrique (éventuellement singuliére) dont la forme de courbure $\xi$ est telle que

$$
\xi=-\sum_{i=1}^{l} n_{i} \delta_{H_{i}}-\eta
$$

oú les $n_{i}$ sont des entiers positifs ou nuls et le symbole $\delta_{H_{i}}$ désigne le courant d'intǵration le long de $H_{i}$. La classe $c_{1}\left(N^{*} \mathcal{G}\right)$ est donc pseudoeffective non triviale (i.e représentée par un courant positif fermé non réduit á zéro) et si l'on reprend l'analyse menée dans [16], ceci montre que $\mathcal{G}$ est en fait minimal; en particulier, $n_{i}=0$ pour tout $i=1, \ldots, l$. 
Par suite, $\eta$ est une métrique lisse á courbure constante négative. Soit $\mathfrak{h}^{\prime} \supseteq \mathfrak{h}$ l'idéal de $\mathfrak{g}$ constitué des champs tangents à $\tilde{\mathcal{G}} ;$ l'algébre de lie résoluble $\mathfrak{g} / \mathfrak{h}^{\prime}$ se redescend via $\pi: \tilde{M} \rightarrow M$ en un faisceau localement constant d'algébre de lie de champs de killing transverses á $\mathcal{G}$. Ce faisceau est en fait réduit á zéro d'aprés la remarque 2.3 et les feuilles de $\mathcal{G}$ sont donc bien fermées.

Ainsi, $\mathcal{G}$ est une fibration holomorphe au-dessus d'une surface de Riemann ; qui plus est, la description de l'algébre de Lie $\mathfrak{h}_{\mathbb{C}}$ nous permet d'affirmer que pour toute feuille $\mathcal{L}$, on a

$$
c_{1}\left(K_{\mathcal{H} \mid \mathcal{L}}\right)=0 .
$$

Il existe par suite un entier naturel $l$ tel que $K_{\mathcal{G}}{ }^{\otimes l}$ soit holomorphiquement trivial; il est en effet facile d'en extraire une section $s$ vérifiant par exemple pour toute feuille $\mathcal{L}$

$$
\sqrt{-1}^{(n-1)^{2}} \int_{\mathcal{L}}(s \wedge \bar{s})^{\frac{1}{l}}=\int_{\mathcal{L}} \theta^{n-1}
$$

oú $\theta$ est une forme de kahler fixée sur $M$.

\section{RÉFÉRENCES}

[1] A.ABOUQATEB, A.EL KACIMI-ALAOUI, Fonctionnelles invariantes et courants basiques, Stud.Mat. 143, (2000) 199-219.

[2] J.ALVAREZ LÓPEZ, Duality in the spectral sequence of riemannian foliations, Am.J.of Maths 111, (1989) 905-925.

[3] A.BEAUVILLE, Variétés Kähleriennes dont la première classe de Chern est nulle, J. Differential Geom. 18, (1983) 755-782.

[4] J.CHEEGER, D.GROMOLL, The splitting theorem for manifolds of nonnegative Ricci curvature, J.Diff.Geom. 6 (1971/72), 119-128.

[5] P.B EBERLEIN, Geometry of nonpositively curved manifolds, Chicago Lectures in Mathematics. University of Chicago Press, Chicago, IL, 1996.

[6] A.EL KACIMI-ALAOUI, Opérateurs transversalement elliptique sur un feuilletage riemannien et applications, Compositio Mathematica 73, (1990) 57-106.

[7] A. EL KACIMI-ALAOUI, B GMIRA, Stabilité du caractère kählérien transverse, Israel J. Math. 101 (1997), 323-347.

[8] A.EL KACIMI-ALAOUI; G.HECTOR, Décomposition de Hodge basique pour un feuilletage riemannien, Ann. Inst. Fourier (Grenoble) 36, (1986), no. 3, 207-227.

[9] S.FRANKEL, Complex geometry of convex domains that cover varieties, Acta Math. 163,(1989) 109-149. 
[10] F.KAMBER, P.TONDEUR, de Rham Hodge theory for Riemannian foliations, Math. Ann. 277 (1987), no. 3, 415-431.

[11] D.LIEBERMAN, Fonctions de plusieurs variables complexes, III (Sém. François Norguet, 1975-1977), Lecture Notes in Math. 670, (1978) 140-186.

[12] X.MASA, Duality and minimality in Riemannian foliations, Comment. Math. Helv. bf 67 (1992), no. 1, 17-27.

[13] P.MOLINO, Riemannian foliations, Translated from the French by Grant Cairns. With appendices by Cairns, Y. Carrière, É. Ghys, E. Salem and V. Sergiescu. Progress in Mathematics, 73. Birkhäuser Boston, Inc., Boston, MA, 1988, 339 pp.

[14] A.M.NADEL, Semisimplicity of the groups of biholomorphisms of the universal covering of a compact complex manifold with ample canonical bundle, Annals of maths 132 (1991), 193-211.

[15] B.SCHIFMAN, A. SOMMESE, Vanishing theorems on complex manifolds, Progress in Math., vol.56, Birkhauser, Boston, 1985.

[16] F.TOUZET, Feuilletages holomorphes de codimension 1 dont la classe canonique est triviale, Ann.Ec.Norm.Sup. 41, (2008) 1-14.

[17] H.H.WU, F.ZHENG, Compact Kähler manifolds with nonpositive bisectional curvature, J.Diff.Geom 61, (2002) 263-287.

[18] S.T.YAU, On the Ricci curvature of a compact Kähler manifold and the complex Monge-Ampère equation. I., Comm. Pure Appl. Math. 31 (1978) 339-411. 